\title{
Notícias sobre os eventos promovidos pela Associação Latino-Americana de Sanscritistas
}

\author{
Lilian Proença de Menezes Montenegro \\ Maria Valíria Aderson de Mello Vargas \\ Universidade de São Paulo
}

Promovidas pelo Curso de Língua e Literatura. Sânscrita do Departamento de Línguas Orientais da Faculdade de Filosofia, Letras e Ciências Humanas da Universidade de São Paulo (FFLCHUSP), realizaram-se, de 11 a 13 de fevereiro de 1980, as CONVERSAÇõES ALAS 80. As reuniões tiveram por objetivo constituir a Associação Latino-Americana de Sanscritistas (ALAS) e eleger sua Diretoria, além de patrocinar a formação de mesas-redondas sobre a história, a metodologia e a bibliografia dos estudos sânscritos na América Latina e a discussão de trabalhos concernentes à cultura sânscrita em geral.

A idéia de constituir uma associação de sanscritistas latinoamericanos surgiu em Weimar, República Democrática Alemã, em maio de 1979, quando lá estiveram, entre aproximadamente 400 especialistas de todo o mundo, participando de IVth WORLD CONFERENCE OF SANSKRIT STUDIES, promovida pela International Association of Sanskrit Studies (IASS), os professores Juan Miguel de Mora e Marja Ludwika Jarocka, ambos da Universidade Nacional Autónoma de México (UNAM), e o Prof. Carlos Alberto da Fonseca, da USP. Pareceu a esses professores que seria bastante proveitosa e importante a formação de uma associação que congregasse os esforços dos especialistas no assunto na América Latina e promovesse a difusão dos estudos sânscritos acadêmicos nos países de fala portuguesa e espanhola por meio da publicação de revistas e boletins e da realização de congressos e colóquios. Escassos e 
mesmo nulos tinham sido até aquele momento os contatos entre especialistas e centros de cultura voltados ao estudo e à divulgação da cultura sânscrita nesses países. A criação da ALAS veio, portanto, preencher uma lacuna que se fazia sentir não só entre os especialistas de um mesmo país, mas também de um país para outro.

De conformidade com o Artigo 5" dos seus estatutos, a ALAS tem por objetivos "promover, diversificar, intensificar, coordenar exclusivamente os estudos sânscritos em todos os países da América Latina; manter contatos com o Centro de Organização dos Congressos Internacionais de Ciências Humanas da Āsia e com a International Association of Sanskrit Studies; organizar colóquios latino-americanos de estudos sânscritos; favorecer as publicações de caráter científico relativas ao estudo da cultura sânscrita; favorecer e facilitar o intercâmbio de experiências acadêmicas entre os seus membros".

São também intenções da ALAS "estabelecer e reforçar os laços entre os centros nacionais latino-americanos de estudos sânscritos; colaborar com os centros superiores de estudos sânscritos na América Latina para a formação de quadros docentes e de pesquisadores; editar revistas e boletins; difundir na América Latina os estudos sânscritos antigos e modernos e, em geral, tudo que contribua para o conhecimento da cultura sânscrita". E, por último, "incentivar o estudo comparativo da cultura sânscrita com fatos culturais particulares a cada uma das culturas latino-americanas; facilitar a atualização bibliográfica dos centros latino-americanos de estudos sânscritos; auxiliar na reciclagem dos docentes e dos pesquisadores do estudo do Sânscrito; constituir e incrementar uma biblioteca especializada em Indologia".

As CONVERSAÇÕES ALAS 80 contaram com a presença, entre outros, dos professores Izidoro Blikstein (responsável, na época, pelo Curso de Língua e Literatura Sânscrita da USP), Carlos Alberto da Fonseca, Mário Ferreira, Lilian Proença de Menezes Montenegro, Maria Valíria Aderson de Mello Vargas, Elisa Fumiko Kikuchi Tamajusuku — todos da USP —, Juan Miguel de Mora e Maria Ludwika Jarocka - da UNAM -, além de alunos e ex-alunos da USP. 
No primeiro dia dos trabalhos, foi criada a ALAS, cuja sede se decidiu instalar em São Paulo, no Curso de Língua e Literatura Sânscrita da USP. Foi eleita, também, a Diretoria para o biênio 1980-1981: Presidente: Prof. Carlos Alberto da Fonseca, Secretário Geral: Prof. Mário Ferreira, Vice-Presidente para o México: Prof. Juan Miguel de Mora, Vice-Presidente para a Argentina: Prof. Fernando Tola (Universidad de Buenos Aires), Secretária Regional para o México: Prof" Marja Ludwika Jarocka, Secretária Regional para a Argentina: Prof ${ }^{a}$ Carmen Dragonetti (Universidad de Buenos Aires). Por decisão unânime da Assembléia, foram nomeados membros honorários, por seu pioneirismo e dedicação aos estudos sânscritos no Brasil, o Reverendo Jorge Bertolaso Stella (falecido em 18-7-80) e a Prof ${ }^{a}$ Maria Luísa Fernandez Miazzi (falecida em 2-11-77) .

No segundo dia do encontro, 12 de fevereiro, realizaram-se as mesas-redondas, nas quais foram debatidas as questões relativas à metodologia do estudo e do ensino da língua e da literatura sânscritas na América Latina. Também nesse dia foram apresentadas as seguintes comunicações: "La relación entre el Pañcatantra y el Coloquio de los perros de Cervantes" (Prof ${ }^{\text {a }}$ Marja Ludwika Jarocka) ; "Acerca de Bhavabhuti y las varnas" (Prof. Juan Miguel de Mora); "Hinduísmo, Lingüística e Semiologia" (Prof. Izidoro Blikstein); "Algumas formalizações narrativas da literatura sânscrita" (Prof. Carlos Alberto da Fonseca); "Cinco poemas de Amaru - uma proposta de tradução" (Prof. Mário Ferreira). Esses trabalhos e os estatutos da ALAS figuram no $1^{\text {? }}$ volume de CULTURA SÂNSCRITA - Alguns aspectos da cultura sânscrita, livro comemorativo à criação da ALAS, publicado pela Universidad Nacional Autónoma de México, em 1980.

No ano seguinte, de 23 a 27 de fevereiro de 1981, realizou-se, na Casa de Cultura Japonesa-USP, a I CONFERÊNCIA LATINOAMERICANA DE SANSCRITISTAS, organizada pelo Curso de Língua e Literatura Sânscrita e pela ALAS. Furam apresentadas, na ocasião, conferências e comunicações, publicadas no $2^{\circ}$ volume de CULTURA SÂNSCRITA - A importância da Língua Sânscrita no passado e na atualidade, da China à América Latina (1981): "La importancia del sanscrito en el pasado y en la actualidad" (Prof. Nicolás Altuchow - Universidad de La República Oriental 
de Uruguay); "Considerações sobre o léxico indiano na Língua Portuguesa" (Prof. Carlos Alberto da Fonseca); "A influência do Sânscrito no Japão" (Prof. Ricardo Mário Gonçalves - USP); "As diáteses do verbo: a questão no indo-europeu e no chinês" (Prof. Mário Bruno Sproviero - USP) ; "O Romance no Sânscrito Clássico" (Prof: Maria Valíria Aderson de Mello Vargas); "Notas para um estudio comparativo entre las diosas Kali y Coatilicue" (Prof ${ }^{a}$ Marja Ludwika Jarocka) ; "Nala e Damayanti: o discurso direto" (Prof Lilian Proença de Menezes Montenegro); "Termos para 'Homem' e 'Mulher' no Rtusamhara de Kalidasa" (Prof. Carlos Alberto da Fonseca); "Agni/Fogo, nos confins da Língua" (Prof. Mário Ferreira); "Literatura clássica comparada: estudo de um texto sânscrito na China do século IV d.C." (Prof ' Terezinha Nakéd Zaratin - USP); "Alguns problemas relativos à grafia das palavras sânscritas" (Prof ${ }^{a}$ Lilian Proença de Menezes Montenegro e Prof. Carlos Alberto da Fonseca); "A terminologia sânscrita do Yoga Clássico: grafia, pronúncia e significado" (Prof. Mário Ferreira). Realizaram-se, ainda, naquela ocasião, um colóquio sobre "Metoơologia para o estudo e o ensino da Língua e da Literatura Sânscritas na América Latina", com a participação dos professores do Curso de Língua e Literatura Sânscrita da USP, do Prof. José Luiz Fiorin (USP) e dos associados, e um curso sobre Bhavabhuti, ministrado pelo Prof. Juan Miguel de Mora. Completando a programação, apresentou-se uma leitura dramática de Abhijñanaçakuntala, acompanhada de um debate sobre "O teatro de Kalidasa".

Promovido pela Universidad Nacional Autónoma de México e pela ALAS, realizou-se, no período de 15 a 21 de fevereiro de 1982, na Cidade do México, a II CONF'ERÊNCIA LATINO-AMERICANA DE SANSCRITISTAS e O I SIMPŌSIO INTERNACIONAL DE LIINGUA SÁNSCRITA. O evento foi inaugurado pelo Dr. Octavio Rivero Serrano, reitor da UNAM e pelo Dr. Fernando Pérez Correa, coordenador de Humanidades da UNAM. Os trabalhos, publicados no $3^{\circ}$ volume de CULTURA SÂNSCRITA - Memória do Primeiro Simpósio Internacional da Língua Sânscrita, constaram das conferências: "Relation of Jiva and Brahman in the Philosophy of Nimbarka" (Prof. Madan Mohan Agrawal - de BanasthaliRajasthan, India); "The Place of the Manduka in a Hindu Cosmolo- 
gical System" (Prof. Ernest Bender - University of Pennsylvania, USA); "Acerca del Tantrismo" (Prof. Juan Miguel de Mora); "Les conditions de la connaissance approfondie du yoga" (Prof. Jean Filliozat - Institute de France); "Kalidasa as a Poet of Aesthetics" (Prof. Rasik Vihari Joshi - El Colegio de México); "Philosophy of perception in India - Nyayasutra 1.1.4." (Prof. Bimal K. Matilal - Oxford University); "Nature of Srauta (Vedic) Sacrifice and Related Terminology" (Prof. H. G. Ranade - Deccan College Postgraduate and Ressearch Institute, India); "The Philosophy of Adaita" (Prof. R. M. Sharma - Panjab University, India) ; "Modern Sanskrit Poetry" (Prof. Satya Vrat Shastri - University of Delhi, India); e das comunicações: "A short study about some Sanskrit syntax facts" (Prof " Maria Valíria Aderson de Mello Vargas); "The Conception of the Mood of the Mind - antahkaranavrti or manovrtti" (Prof. M. M. Agrawal - India); "Profesor Doctor Stefan Stasiak (1884-1962) y su publicación del Relato sobre la India de un viajero polaco en el siglo XVI" (Prof. Nicolás Altuchow - Uruguay); "Perspective on Dominance: The Role of Psychomagical means (UPAYAS) of Diplomacy and War" (Prof. Shiva G. Bajpai - California State University, USA) ; "La Signification du Madhyamaka, Reconsidération des arguments de Nagarjuna contre le mouvement" (Prof. Kamaleswar Bhattacharya - Centre National de la Recherche Scientifique, France); "Voir le brahman: un mirage sémiologique. Pròblemes de traduction de la Kena Upanisad" (Prof. Izidoro Blikstein - USP) ; "The British Reception of Sir William Jone's Translation of the Sakuntala" (Prof. Garland Cannon - Texas A\&M University, USA) ; "A composição nominal em Sânscrito: rumo à imaginação poética" (Prof. Carlos Alberto da Fonseca) ; "Catustava (Niraupamya, Lokatita, Acintya, Paramartha) de Nagarjuna" (Prof: Carmen Dragonetti - Consejo Nacional de Investigaciones Científicas y Técnicas, Argentina); "Pañcha-vrttinispadyamanam sanskritbhasa-saunddrayam or Beauty of Sanskrit language as caused by five vrtti's i.e. the grammatical functions" (Prof. Bhagavatprasad Padya - Gujarat University, India); "The Interrogative Pronoun in Sanskrit and Early Brajabhasa" (Prof. Gilbert Pollet - University of Leuven, Bélgica); "Vedic Religion and its Indo-european Background" (Prof. Edgar Charles Polomé - 
University of Texas at Austin, USA) ; "Krsna as The Eighth Child" (Prof. Benjamin Preciado Solís - El Colegio de México) ; "O episódio de Nala no Mahabharata: algumas notas para uma análise semiótica de um texto sânscrito épico - Cantos I, II e III" (Prof Lilian Proença de Menezes Montenegro) ; "Naisadha Padalalityam" (Prof. Mukunda Madhava Sharma-Gauhati University, India); "Drstisrstivada - an analysis and critical appraisal" (Prof. Ram Murti Sharma - Panjab University); "Tantra and Veda in Hindu Cultural Context" (Prof. Lalan Prasad Singh — Magadh University, India); "On the Indian Concept of the Body" (Prof. Frits Staal University of California, USA); "Tres aspectos del atman en la Brhadaranyaka-Upanisad" (Prof. Fernando Tola - Consejo Nacional de Investigaciones Científicas y Técnicas, Argentina); "La India: sus creencias filosófico-religiosas a través de la obra de Onesícrito" (Prof Rosália C. Vofchuk - Consejo Nacional de Investigaciones Científicas y Técnicas, Argentina); "On the meaning of jana - in the RV" (Prof. Stefan Zimmer - Freie Universität, Berlin, Germany).

Naquela oportunidade, realizou-se a Assembléia Geral da ALAS, quando se deu a eleição da nova Diretoria para o biênio 82-83, tendo sido reeleito presidente o Prof. Carlos Alberto da Fonseca, Secretária Geral: Prof ${ }^{a}$ Marja Ludwika Jarocka, Vice-Presidente para o México: Prof. Juan Miguel de Mora, Vice-Presidente para a Argentina: Prof. Fernando Tola, Secretária Regional para o Brasil: Prof Maria Valíria Aderson de Mello Vargas, Secretária Regional para a Argentina: Prof Carmen Dragonetti. Durante a Assembléia foi aprovada a criação da Comissão Bibliográfica da ALAS, composta pelos Professores Izidoro Blikstein e Lilian Proença de Menezes Montenegro.

Nas atividades sociais do Congresso, os participantes foram brindados com programas de danças folclóricas mexicanas a cargo do Departamento de Danza de la Dirección General de Difusión Cultural de la UNAM e fizeram visitas às escavações do Templo Mayor, ao Museu Nacional de Antropologia e à zona Arqueológica de Teotihuacán. Também puderam apreciar uma apresentação teatral, Mansa-Puspam (Flor de Carne), baseada nos textos do $R g$ Veda "Indrani y el Mono Macho (X. 86)", tradução de Fernando Tola, e "Pururavas e Urvaci (X. 95)", tradução de Juan Miguel de Mora. 
No período de 2 a 10 de fevereiro de 1983, tiveram lugar, no Prédio de Ciências Sociais da FFLCH da USP, as CONVERSAÇôES ALAS 83, ocasião em que foram apresentados os trabalhos: "A contribuição do Sânscrito para a semântica indo-européia: estudo do campo morfo-semântico de kapa, "cabeça"' (Prof. Izidoro Blikstein) ; "Comunicaciones entre la India y Occidente en la Antigüedad" (Prof' Marja Ludwika Jarocka) ; "O 'sentimento da gramaticalidade' na língua sânscrita: algumas palavras sobre o pensar analógico sintético" (Prof. Carlos Alberto da Fonseca) ; "Considerações sobre o léxico do jogo de dados no Sânscrito" (Prof "Lilian Proença de Menezes Montenegro); "Algunos aspectos de la cultura sánscrita" (Prof. Juan Miguel de Mora). Esses trabalhos serão publicados no $4^{\circ}$ volume de CULTURA SÂNSCRITA.

Em junho de 1984, de 13 a 15, na Faculdade de Letras da Universidade de São Paulo, realizou-se, com o apoio da Fundação de Apoio à Pesquisa do Estado de São Paulo (FAPESP) e da Coordenadoria de Atividades Culturais da USP (CODAC), a III CONFERENCIA LATINO-AMERICANA DE SANSCRITISTAS com o tema geral Obras-chave da Literatura Sânscrita, de cujo programa constaram os trabalhos: "Influências do Rgveda no Budismo Japonês: - rito de Homa" (Prof. Ricardo Mário Gonçalves); "Nala $e$ Damayati: a tradução latina de Franz Bopp" (Prof. Izidoro Blikstein) ; "Efeitos de harmonia e de sugestão no romance Daçakumaracarita, de Dandin" (Prof " Maria Valíria Aderson de Mello Vargas); "Sugestões para uma leitura do Rtusamhara, de Kalidasa, como poema erótico" (Prof. Carlos Alberto da Fonseca); "O ensino do sânscrito na Universidade Federal do Ceará" (Prof. José Rebouças Macambira - Universidade Federal do Ceará) ; "A figurativização do feminino. Considerações acerca do figurativo em alguns relatos do Mahabharata" (Prof ${ }^{a}$ Lilian Proença de Menezes Montenegro); "O doutrina do renascimento em Rgveda I.164" (Prof. Roberto Martins - Universidade de Campinas); "Os avataras de Visnu: relatório de um exercício didático de tradução poética" (Prof. Mário Ferreira e Maria Regina de Souza - USP). Os trabalhos, reunidos nos Anais da III CONFERENCIA LATINO-AMERICANA DE SANSCRITISTAS (USP, 1984), deverão compor o $5^{\circ}$ volume de CULTURA SÂNSCRITA. 
No período de 22 a 25 de abril de 1985, na Casa da Cultura Japonesa-USP, teve lugar a SEMANA DA INDIA (CONVERSAÇÕES ALAS 85), com o apoio da CODAC, ocasião em que foram apresentadas as conferências: "Sociedade e cultura na India" (Prof. Eduardo J. Barros - Universidade Estadual de Londrina); "Pintura Indiana" (Prof. Mário Ferreira) ; "Octavio Paz e a India" (Prof ${ }^{a}$ Maria Lúcia Fabrini de Almeida); "A religiosidade popular da Îndia" (Prof. Carlos Edgar Kugelmas); "Os quatro açramas dos brâmanes" (Prof. Roberto Martins) ; "Literatura épica sânscrita" (Prof ${ }^{a}$ Lilian Proença de Menezes Montenegro, Márcia Klimiuc e Suzanne Fortenberry Rodrigues) ; "Teatro indiano clássico" (Prof. Carlos Alberto da Fonseca); "Fábula, Conto e Romance na Cultura Sânscrita" (Prof a Maria Valíria Aderson de Mello Vargas) ; "Idéias sócio-econômicas do budismo indiano" (Prof. Ricardo Mário Gonçalves). Esses trabalhos deverão ser publicados no $6^{\circ}$ volume de CULTURA SĀNSCRITA.

Quanto às atividades sociais do evento, realizou-se, no auditório da Faculdade de Arquitetura e Urbanismo da USP, um Recital de Sitar, com Alberto Marsicano, e uma leitura dramática demonstrada da peça do teatro sânscrito clássico, Xacuntalá reconhecida, de Kalidasa, pelo grupo Bharatanatyadarpana.

Em 1988, com a promoção do Curso de Língua e Literatura Sânscrita da USP e com o apoio da FFLCH-USP, da ALAS e da Sociedade Brasileira de Estudos Clássicos, realizou-se o Ciclo de Debates sobre a İndia Antiga, no Conjunto Didático de Antropologia, Ciência Política, Filosofia e Sociologia, subdividido nas seguintes sessões: dia 24 de março — "Ordem e Desordem. no pensamento da India Antiga", com as conferências: "O Conceito de Desarmonia na Cosmologia Védica" (Prof. Mário Ferreira), "Dharma e Adharma na Narrativa Épica" (Prof" Lilian Proença de Menezes Montenegro), "A ética das máximas no fabulário sânscrito" (Prof ${ }^{a}$ Maria Valíria Aderson de Mello Vargas), "O 'Bem-feito' e o 'Mal-feito' na Estética Sânscrita” (Prof. Carlos Alberto da Fonseca) ; dia 6 de maio - "Alguns Aspectos da Condição da Mulher na India Antiga", com as conferências: "A mulher na India Antiga: Quando se Apaga a Brasa do Incenso" (Prof. Carlos Alberto da Fonseca), "Convenções Sociais e 'Figura' Feminina no Discurso 
Mítico-Religioso e sua Expressão na Literatura Ėpica Sânscrita". (Prof $f^{a}$ Lilian Proença de Menezes Montenegro), "As Diversas Feições do Comportamento da Mulher Observadas nos Textos da Literatura Sânscrita Clássica" (Profa Maria Valíria Aderson de Mello Vargas), "Virgem e Cortesã: O Ser Feminino Segundo a Concepção Tântricà" (Prof. Mário Ferreira); dia 31 de maio: - "Alguns Aspectos da Reflexão Lingüístico-Literária na India Antiga", com as conferências: "Alguns Aspectos da reflexão linguística na poesia arcaica da India Antiga" (Prof. Mário Ferreira), "Aspectos da figuratividade nos textos épicos do Mahabharata. Algumas Considerações" (Prof Lilian Proença de Menezes Montenegro), "Efeitos de Harmonia e de Sugestão no Romance Daçakumarácarita, de Dandin" (Profa Maria Valíria Aderson de Mello Vargas), "O 'sentimento da gramaticalidade' na língua Sânscrita: algumas palavras sobre o 'pensar analógico sintético" (Prof. Carlos Alberto da Fonseca). . Esses trabalhos encontram-se em fase de publicação na coleção BHARATA a ser editada pela Universidade de São Paulo.

Em outubro e novembro de 1988, a ALAS e o Curso de Língua e Literatura Sânscrita da USP, com o apoio da FFLCH-USP, da FAPESP e da Comissão de Cooperação Internacional da USP, promoveram as seguintes atividades: a) O Ciclo de Conferências "Relações culturais entre a İndia e o Ocidente", proferido pela Prof Marja Ludwika Jarocka (UNAM), e subdividido em 3 temas: "Influência do Pancatantra em El Casamiento engañoso e El Coloquio de los perros, novelas exemplares de Miguel de Cervantes", "Influências da India em El Mono Gramático de Octavio Paz" e "Relações da India com o mundo ocidental antes e depois de Alexandre o Grande"; b) O Curso de Difusão Cultural "A cultura sânscrita e sua contribuição para a cultura universal", ministrado pelo Prof. Juan Miguel de Mora (UNAM), na qualidade de Professor-Visitante, que desenvolveu os seguintes temas: "Considerações sobre a história e a cultura da India", "Alguns aspectos do Rgveda", "Tradição e continuidade do teatro sânscrito — do periodo védico ao clássico", "O zero, o sistema decimal, a astronomia e outras contribuições da India para a matemática universal", "O Ayurveda: a medicina antiga da India, seus progressos, sua influência sobre a medicina grega (e sobre a européia a partir do século XVIII)", "O Yoga tântrico: o 
psico-somático na India", "Influências de idéias tântricas na Espanha e no México. Seitas de inspiração hindu nos séculos XVI e XVII", "O princípio dos contrários na literatura sânscrita", "O conto hindu: Pañcatantra, Hitopadeça, Os trinta e dois relatos do trono, As histórias do Vetala, etc.". Na ocasião, o Prof. Mora doou gentilmente ao acervo da área de Língua e Literatura Sânscrita da USP duas importantes obras de sua autoria, a saber, a tradução de El último lance de Rama, de Bhavabhuti, publicada pelo Instituto de Investigaciones Filológicas da Universidad Nacional Autónoma de México em 1984, e La Dialéctica en el Rig Veda, publicada no México pela Editorial Diana em 1978.

Os objetivos iniciais que constituiram as bases da ALAS ultrapassaram, sem dúvida, as primeiras expectativas dos membros fundadores. Ao mesmo tempo em que se processou a integração dos sanscritistas latino-americanos, a Associação conseguiu projetar, no cenário do sanscritismo internacional, os estudos sânscritos por eles realizados. 